\title{
Treatment of Chronic Temporomandibular Joint Pain and Sleep Disordered Breathing by Teledontic and Telegnathic Protocol Utilizing Total Joint Replacement
}

\author{
Joseph Yousefian', Michael N. Brown'1, Samuel L. Bobek², Norman C. Chiang1, Ryan Y. Chiang1 \\ ${ }^{1}$ Independent Researcher, Bellevue, WA, USA \\ ${ }^{2}$ Swedish Hospital, Bellevue, WA, USA \\ Email: joseph@dryousefian.com
}

How to cite this paper: Yousefian, J., Brown, M.N., Bobek, S.L., Chiang, N.C. and Chiang, R.Y. (2017) Treatment of Chronic Temporomandibular Joint Pain and Sleep Disordered Breathing by Teledontic and Telegnathic Protocol Utilizing Total Joint Replacement. Open Journal of Orthopedics, 7, 308-319.

https://doi.org/10.4236/ojo.2017.710031

Received: August 27, 2017

Accepted: October 13, 2017

Published: October 16, 2017

Copyright (๑) 2017 by authors and Scientific Research Publishing Inc. This work is licensed under the Creative Commons Attribution International License (CC BY 4.0).

http://creativecommons.org/licenses/by/4.0/

\begin{abstract}
Orthopedic clinicians frequently encounter patients with temporomandibular joint (TMJ) pain and associated sleep disordered breathing (SDB) that coexists with the patient's orthopedic conditions. The systemic effects and associated comorbidities caused by TMJ and associated SDB are commonly not recognized as potential contributors to the patient's long-term orthopedic outcome. This article describes a comprehensive and interdisciplinary medical dental treatment, which was able to successfully address patient's severe chronic TMJ, head, neck and shoulder pain as well as other health concerns including SDB. Moreover, a new teledontic and telegnathic treatment protocol and principles utilizing total joint replacement for care of patients with chronic TMJ pain and SDB will be introduced describing a completed case.
\end{abstract}

\section{Keywords}

Sleep Disordered Breathing, Upper Airway Resistance Syndrome, Temporomandibular Joint, Pharyngorofacial Disorders, Teledontics, Telegnathic Surgery, Total Joint Replacement

\section{Introduction}

TMJ disorders (TMJD) as part of masticatory disorders (MD), most often but not always characterized by painful musculoskeletal signs and symptoms in the masticatory and associated structures in head, neck and shoulder area, estimated to affect approximately $5 \%$ to $15 \%$ of the population, predominantly women [1] [2] [3] [4]. 
The TMJD includes internal derangement, osteoarthritis, chronic recurrent dislocation, ankylosis, neoplasia and infection [5].

Subjective sleep disturbance has been consistently reported in MD patients [4] [6] [7]. A recent investigation employing polysomnogram (PSG) recordings and clinical sleep interviews in a sample of MD cases showed that MD is associated with primary sleep disorders, such as insomnia and obstructive sleep apnea (OSA): nearly $36 \%$ of MD cases met diagnostic criteria for insomnia, and over $28 \%$ met criteria for OSA [8]. In the same sample of MD cases, sleep efficiency on PSG was directly related to a measure of pain threshold, suggesting an association between pain and sleep disturbance in MD [8]. Additionally, a high prevalence of MD was reported in clinical patients with mild to moderate OSA referred for a clinical dental evaluation, lending further support to the association between MD and OSA [9]. It was argued that a $28 \%$ rate of OSA diagnosis in a sample of MD patients composed mainly of young females with relatively low body mass index (BMI) lends support to the possibility of elevated risk of OSA in $\mathrm{MD}$, requiring further evaluation in a large scale study [8].

Further, in this sample of MD cases, the mean apnea-hypopnea index (AHI) was only borderline at 5.1 events per hour [8] [10]. While the diagnosis of OSA in these participants was based on established criteria for scoring and interpreting the respiratory disturbance index (RDI) using standard laboratory PSG, which includes respiratory effort related arousals (RERAs) in addition to apneas and hypopneas [11] [12], the degree of sleep disordered breathing among MD cases who met the criteria for OSA was predominantly mild [8] [10].

Chronic TMJ, head, neck and shoulder pain can be a debilitating condition. On the other hand, SDB can be a life-threatening disease. Obstructive sleep apnea syndrome (OSAS) is considered a more severe form of SDB and can have profound systemic effects that can affect orthopedic outcomes such as neck and shoulder instability and pain. Since the OSAS has been recognized as a major public health problem, many efforts have been made to define and understand this syndrome [13].

Breathing abnormalities during sleep are not limited to patients exhibiting typical obstructive apnea but include a continuum of events from simple snoring to OSAS that constitute a group of diseases named SDB. The upper airway resistance syndrome (UARS) as mild form of SDB was initially reported by Guilleminault et al. in 1993 [14]. This particular syndrome has been described based on the hypothesis that repetitive increases in respiratory efforts that are inducing arousals known as RERA might produce a significant disease leading to daytime sleepiness and functional symptoms and are associated with cardiovascular and cognitive morbidities [14].

Potential comorbidities of URAS include; nocturia, nocturnal awakening, cognitive impairment, headaches, vasomotor rhinitis, irritable bowel syndrome, psychological complaints such as difficulty in concentrating and depressive mood, sleep inefficiency, 75\% increased chance of motor vehicle accidents attributable to excessive daytime sleepiness, carotid atherosclerosis, increase in sym- 
pathetic outflow, hypertension, cardiovascular and metabolic consequences [15] [16] [17].

Many of the etiologies that contribute to these disorders are unrecognized by clinicians dealing with these patients and one can miss an opportunity to recognize and screen for potential reversible causes of these conditions.

These recognizable etiologic factors involve oral, dental, temporomandibular joint, pharyngeal and facial bone structures, which are referenced hereinafter as pharyngorofacial disorders (POFD). Having a basic understanding of the POFD that can be at the root cause of TMJ and sleep breathing disorders may allow physicians to identify underlining structural and functional pathology that is having an unrecognized profound effect on the oral and systemic health.

The data published by American Academy of Sleep Medicine on September 2014 , estimated an increase in prevalence of SDB to 26 percent amongst adults between the ages of 30 and 70 years and gave much credit to the rising obesity epidemic [18]. Although Franklin et al. investigated 400 females from a population based random sample of 10,000 Swedish females and OSA (apnea/hypopnea index (AHI) $\geq 5$ ) was found in 50\% (95\% CI 45\% - 55\%) of females aged $20-70$ yrs. Severe sleep apnea (AHI $\geq 30$ ) was present in $14 \%$ (95\% CI $8.15 \%-21 \%)$ of females aged 55 - $70 \mathrm{yrs}$ and in $31 \%$ (95\% CI 12\% - 50\%) of obese females with a body mass index of $\geq 30 \mathrm{~kg} \cdot \mathrm{m} \mathrm{(}-2)$ aged 55 - $70 \mathrm{yrs}$ [19]. Asian men with severe obstructive sleep apnea have less tendency to be obese, but may present with significant posterior airway restriction leading to OSA [20].

Besides obesity there are other abnormalities in anatomy and neuromuscular function of upper airway, which clinicians need to recognize that are more important risk factors [21] [22] [23]. POFD, including hypoplastic maxilla and mandible, is common findings in SDB including OSA as well as URAS and needs to be recognized by clinicians [18]. POFD and its effects on the systemic health continue to be underestimated in the development and severity of SDB [23] [24].

Orthopedic practitioners have great opportunity in screening for the presence of SDB and OSAS as a complex health conditions contributing in development of other systemic issues such as metabolic syndrome, obesity and musculoskeletal disorders [25].

Every pound of body weight places four to six pounds of pressure on each knee joint. Osteoarthritis (OA) - a progressive "wear and tear" disease of the joints—is frequently associated with obesity [26].

A narrowed upper airway often is typically a component of SDB and OSA which can lead to postural compensation [27]. Most of these patients will try to increase the airway patency by altering the craniocervical posture during the daytime activities or sleep [28]. The anterior head carry position can lead to a number of postural aberrations that can lead to effects in the upper cervical spine, lower cervical spine, shoulders as well as lumbar spine and pelvis (Figure 1) [27]. 

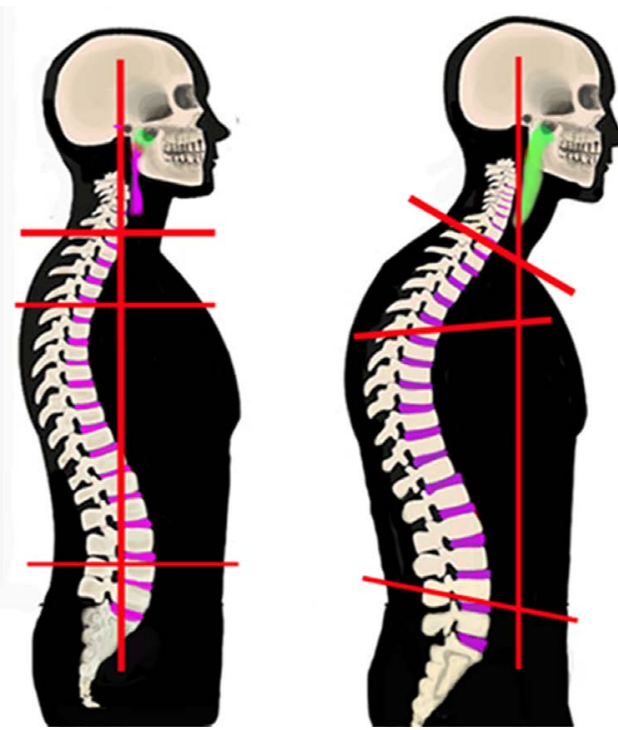

1. Forward head tilt shifting center of gravity.

2. Head thrust help open the airway.

3. Upper body weight is shifted backward.

4. Hips are shifted forward.

5. Lumbar loardosis is increased.

6. Increased facet joint loading in upper cervical spine and cervicothroacic junction.

Figure 1. Schematic drawing showing the postural changes in SDB and OSA patients.

The case presented in this paper demonstrates the effective participation of the orthopedic physician and dental practitioner equipped with teledontics and telegnathic knowledge as a member of an interdisciplinary dental/medical team collaborating in the treatment of SDB and chronic TMJ, head, neck and shoulder pain by treatment of POFD. This article also demonstrates the effectiveness of teledontics and telegnathic surgery utilizing total joint replacement procedure in the treatment of SDB [24].

\section{Case Report}

A 53-year old female was referred for evaluation with the chief complaint of severe snoring disturbing others, jaw clicking-popping, TMJ pain, headaches, neck and shoulder pain. Clinical examination showed a Class II Division 1 malocclusion combined with retrognathic position of maxilla and mandible (Figure 2), and also revealed a significant condylar remodeling of both TM joints that caused severe intracapsular pain on both sides (Figure 3). Patient also disclosed a long-term history of excessive daytime sleepiness, fatigue, morning headaches and discomfort in the area of temporal and masseter muscles. Patient had previous history of orthodontic treatment, three times including extraction of four bicuspids with use of headgear during her youth, further treatments in combination with lower jaw orthognathic surgery in adulthood and later another nonsurgical correction of the bite with significant relapse.

The treatment was initiated by the application of centric relation (CR) repositioning splint combined with physical therapy, soft diet, daily oral use of $100 \mathrm{mg}$ of Doxycycline and $20 \mathrm{mg}$ Feldene as anti-inflammatory medications. Patient's pain and discomfort although reduced in response to this treatment but never completely resolved. 

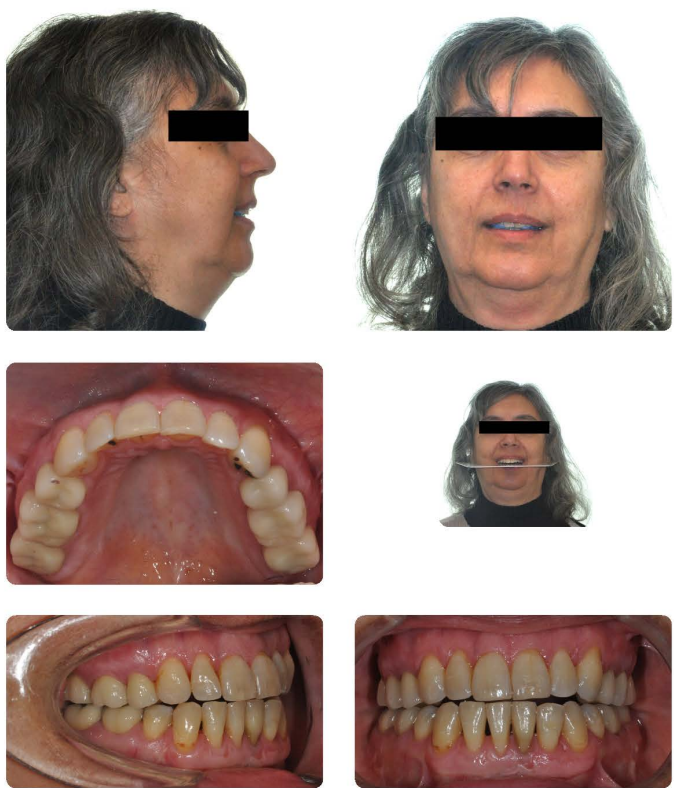
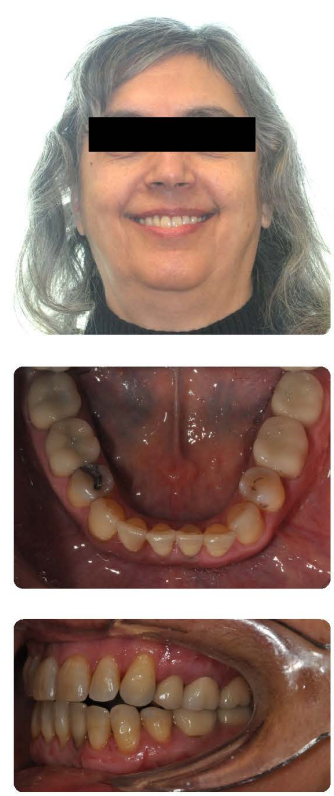

Figure 2. Pre-treatment extraoral and intraoral photographs.

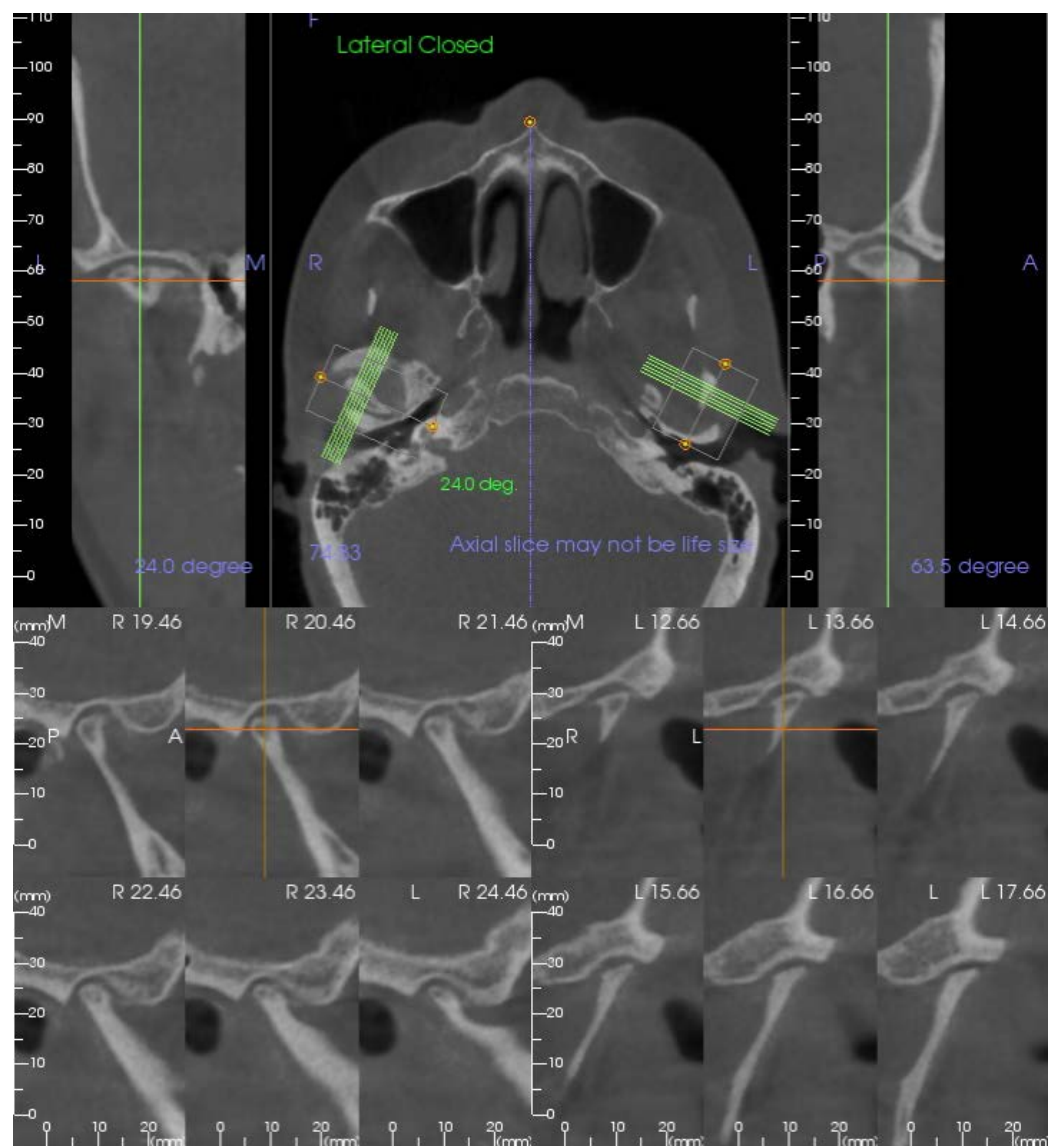

Figure 3. Pre-treatment CBCT of the TM joints.

This treatment protocol improved the severity of the condition but the TMJ and intracapsular pain and discomfort never entirely eliminated. 
Due to progressive hypoplastic remodeling of the condyles, patient's open bite and retrognathic position of the mandible continued to increase.

The patient reported more severe snoring, nocturnal bruxism and daytime sleepiness. Based on these developments, patient was referred for in lab sleep study.

The sleep study revealed that the patient had mild OSA with apnea hypopnea index (AHI) of 6.8 and upper airway resistance syndrome (URAS) with respiratory disturbance index (RDI) of 31.5 and lowest blood oxygen desaturation of $91 \%$. The sleep study also demonstrated significant nocturnal bruxism associated with arousals. At this stage, the patient was advised to use continuous positive air pressure (CPAP) with $7 \mathrm{CM}$ of water pressure for improvement of the OSA. Regular use of CPAP in combination with CR splint improved the SDB and also considerably reduced daytime fatigue and sleepiness. She reported absence of her sleep bruxism and improvement of TMJ symptoms although some pain, clicking and TMJ discomfort persisted upon awakening in the morning and during the day.

There is some evidence suggesting that obstructive sleep apnea is associated with sleep bruxism. Thus, in patients with concomitant obstructive sleep apnea and sleep bruxism, most sleep bruxism events occurred after apnea-hypopnea events, suggesting that sleep bruxism events occurring close to apnea-hypopnea events is a secondary form of sleep bruxism [29] [30]. Further evidence suggest that myofascial pain in TMJ and POF disorders is associated with mild elevation in sleep fragmentation and increased frequency of RERA events [4].

At meanwhile patient's husband was appointed to a new job in Pakistan. Based on these developments and possible lack of access to effective treatment after relocation including access to uninterrupted electricity for use of CPAP, patient requested treatment plan with more predictable results for reduction of accompanying symptoms including presence of chronic pain and excessive daytime sleepiness and fatigue.

The alternative treatment plan was proposed including the counterclockwise maxillomandibular advancement (CC-MMA) as teledontic/telegnathic protocol to correct the retrusive position of the maxillofacial components to improve the occlusion and facial skeletal discrepancies. Another anticipated goal was to improve the existing SDB by significant expansion of POF complex.

When orthodontic and orthognathic surgical procedures are used for the expansion and restoration of the POF complex for treatment of SDB and OSA, they are referred to as teledontics and telegnathic surgery [24] [31] [32]. This treatment protocol avoids the use of procedures and techniques, which can retract or constrict the jaws or dentition including; extraction of teeth, use of headgear or functional appliances that in long run impacting the upper airway negatively [33] [34].

Based on persistence intracapsular joint pain in addition to the increased chance of post surgery relapse due to severe hypoplastic remodeling of the TMJ condyles, patient elected another alternative plan. This included CC/MMA sur- 
gery in combination with bilateral total joint replacement as teledontic/telegnathic protocol to address her chief complaints (Figure 4) [35] [36].

\section{Treatment Results}

A balanced facial profile with improved chin protrusion was obtained (Figure 5) but, most importantly, the patient reported a significantly improved level of alertness and absence of TMJ pain, headaches, neck and shoulder symptoms. Her spouse reported almost no disturbances during sleep, and the postsurgical polysomnography showed an AHI of 2.7 and RDI of 2.8 and lowest blood oxygen saturation of $93 \%$, which is normal. A remarkable increase in the retropalatal, retroglossal, as well as hypoglossal airway spaces concomitant with maxillary and mandibular advancement was evident in pre and post-treatment cephalometric radiograph (Figure 6).

\section{Discussion}

The most favorable care for patients with SDB is treatment provided by an interdisciplinary team that includes members from the appropriate dental and medical disciplines. Prescribed therapies might include weight loss, behavior modification, oral appliances, soft-tissue surgery, skeletal surgery, or some combination thereof [25] [37].

The field of dentistry and its involvement with the POF environment, provides the dental practitioner with an opportunity in screening for the presence of SDB and OSA as a complex health condition or participation in its treatment [24].

Teledontics and telegnathic treatment protocol is the science and technologies for diagnosis, prevention, management and treatment of SDB by means of expansion, restoration and maintenance of POF complex [24] [31] [32].
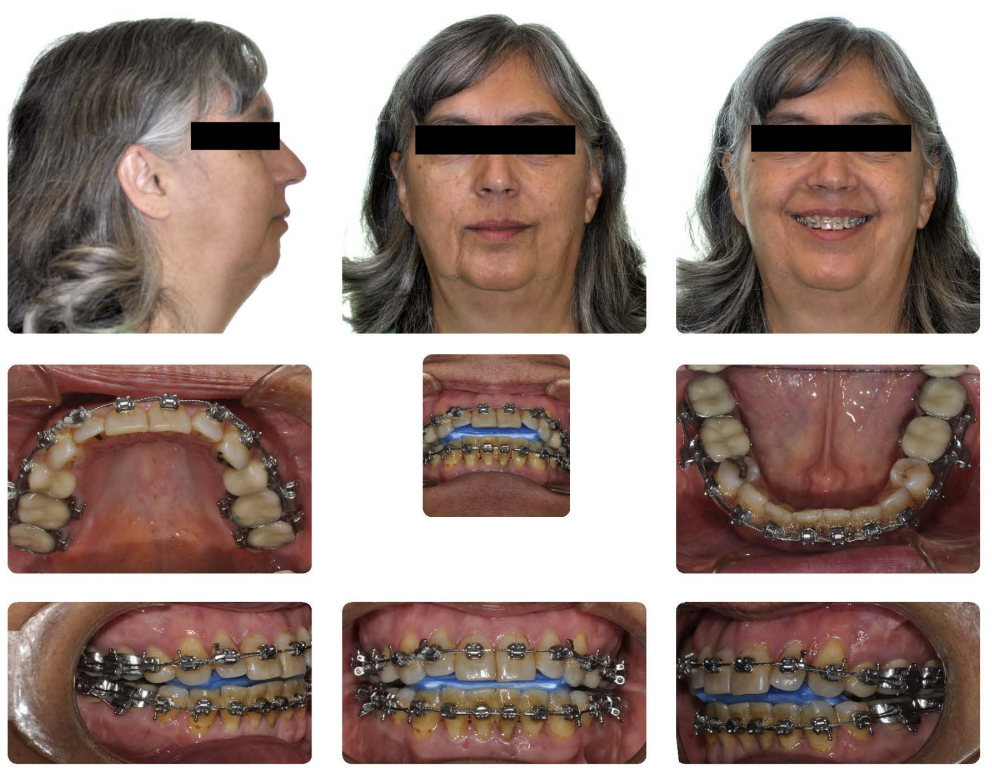

Figure 4. Pre-telegnathic surgery extraoral and intraoral photographs. 

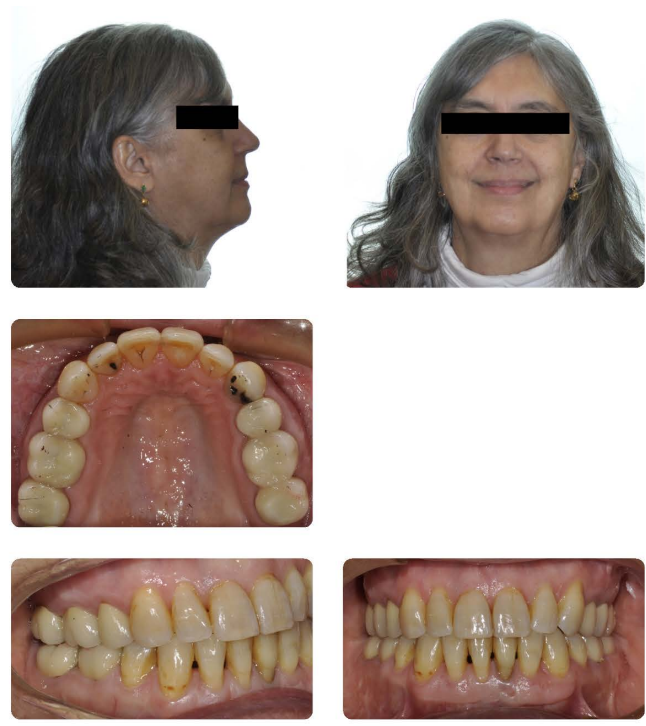
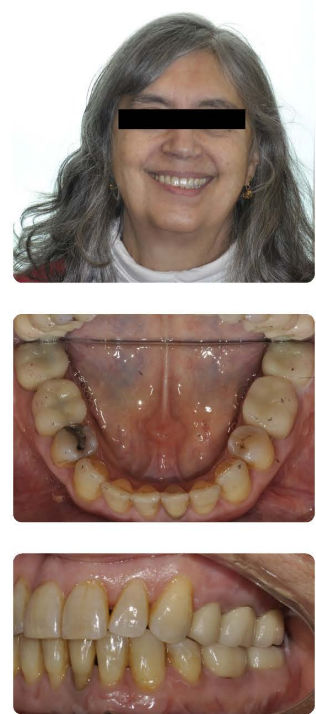

Figure 5. Post-treatment extraoral and intraoral photographs.
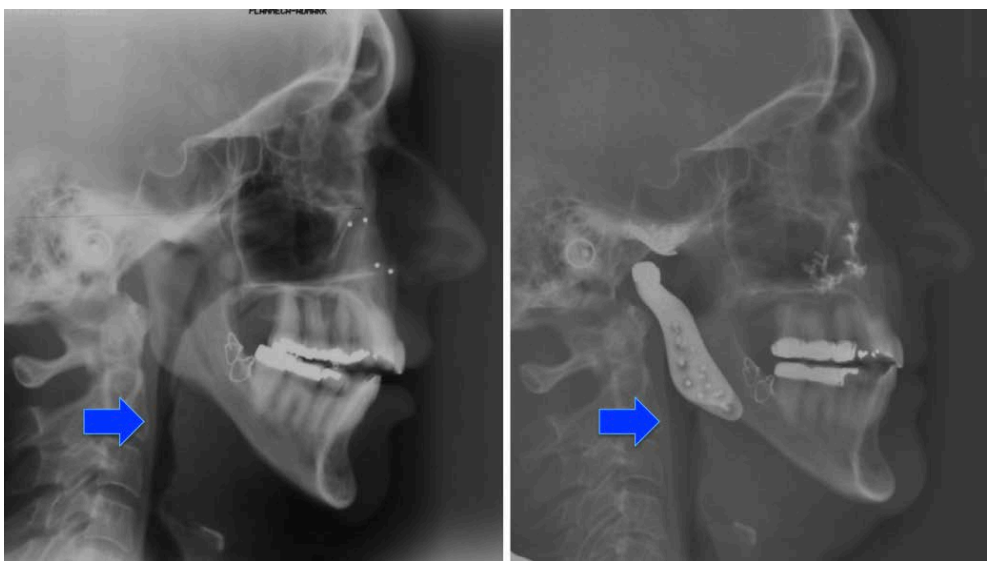

Figure 6. Pretreatment and post-treatment lateral view cephalometric radiographs (Arrows point to the enlargement of the airway before and after treatment).

CC/MMA as teledontic/telegnathic protocol by expansion of the posterior nasopharyngeal opening also augment the nasal cavity. A nasal cavity volume increase should reduce the resistance to nasal airflow [38]. If the upper airway is considered a simple tube, as the radius of the tube increases, the resistance to flow decreases exponentially to the fourth power (resistance $=8 \mathrm{L \eta} / \pi \mathrm{r} 4$ ) [39]. Therefore, even small increases in the diameter of the tube can dramatically decrease the resistance to nasal or pharyngeal air flow [39].

Nonsurgical approach including the retraction of the upper dentition would have addressed the orthodontic aspects of this patient's malocclusion. Although correction of the malocclusion was not stable in the past and only was one of the objectives of the treatment, overshadowed by SDB, as a more health threatening condition.

Constricting the dental arches and upper airway due to retraction of upper 
teeth, often occurs with orthodontic treatment procedures such as four bicuspid extraction, use of headgear or functional appliances [33] [34] could be an obstacle to optimally improving the patient's pharyngorofacial health and overall wellbeing [25] [37]. Making already constricted arches smaller anteroposteriorly not only can potentially exacerbates the symptoms and complications of SDB [25] [37] but also decline the facial and dental esthetic [40] [41] [42] [43] [44]. Therefore, any orthodontic as well as orthognathic treatment modalities including retraction or impaction of posterior maxillary structures to correct the bite discrepancies in this potentially vulnerable population of patients should be reconsidered [37] [45]. Ideally, a treatment plan involving expansion of the POF complex in all three dimensions would be more effective in treating these patients [24] [31] [35].

This paper helps the readers to understand the interrelation of POFD including TMJD and development of SDB. Also, a comprehensive and interdisciplinary medical and dental treatment planning that was able to successfully address the patient's health issues including chronic TMJ as well as cervical pain and sleep disordered breathing.

\section{Conclusions}

Orthopedic specialist and subspecialty physicians commonly encounter patients with multiple orthopedic and chronic pains who also suffer from other comorbidities of SDB and OSA.

Physicians evaluating patients with TMJ pain, maxillofacial pain and cervical spine complaints as well as SDB and OSA should obtain a medical history that includes a basic dental history and sleep history to unmask possible POFD as a cause or contributing factor. Physicians can rapidly learn the basic screening tools necessary to identify possible POFD and make appropriate referrals to physicians and dentist with special training and expertise in teledontic dentistry and telegnathic surgery.

Physicians unaware of this link between previous dental and orthodontic treatment and the associated POFD that can arise from this treatment will miss an opportunity to identify contributing factors to the patient's clinical presentation.

There is a pandemic of SDB and OSA in both pediatric and adult population globally with a significant medical and socioeconomic impact. Physicians should be aware of an evolution of understanding in regard to impact of POF discrepancies that can play a critical role in the development of these conditions and its impact on systemic health of patients.

While oral surgeons are moving away from "orthognathic" to a "telegnathic" approach which reflects the forward positioning of the maxilla and mandible for the treatment of SDB, orthodontists with an understanding of a "teledontic" approach will be able to prevent and treat SDB in pediatric and adult patients by proper expansion of the POF complex, avoiding extractions, use of headgear or functional appliances. This approach could prevent the development of SDB in 
our pediatric population and treat the underlying cause in those patients presenting with OSA. This provides an interdisciplinary approach to managing not only the orthopedic patient, but addressing many of the contributing factors that may be root causes that led to the development of the orthopedic clinical presentation.

\section{References}

[1] Janal, M.N., Raphael, K.G. and Nayak, S. (2008) Prevalence of Myofascial Temporomandibular Disorder in US Community Women. Journal of Oral Rehabilitation, 35, 801-809. https://doi.org/10.1111/j.1365-2842.2008.01854.x

[2] Isong, U., Gansky, S.A. and Plesh, O. (2008) Temporomandibular Joint and Muscle Disorder-Type Pain in U.S. Adults: The National Health Interview Survey. Journal of Orofacial Pain, 22, 317-322.

[3] Bagis, B., Ayaz, E.A. and Turgut, S. (2012) Gender Difference in Prevalence of Signs and Symptoms of Temporomandibular Joint Disorders: A Retrospective Study on 243 Consecutive Patients. International Journal of Medical Sciences, 9, 539-544. https://doi.org/10.7150/ijms.4474

[4] Dubrovsky, B.L.G. (2014) Polysomnographic Investigation of Sleep and Respiratory Parameters in Women with Temporomandibular Pain Disorders. Journal of Clinical Sleep Medicine, 15, 195-201.

[5] De Sena, F.M., de Mesquita, K.S.F. and Santos, F.R.R. (2013) Prevalence of Temporomandibular Dysfunction in Children and Adolescents. The Revista Paulista de Pediatria, 31, 538-545. https://doi.org/10.1590/S0103-05822013000400018

[6] Yatani, H., Studts, J. and Cordova, M. (2002) Comparison of Sleep Quality and Clinical and Psychologic Characteristics in Patients with Temporomandibular Disorders. Journal of Orofacial Pain, 16, 221-228.

[7] Quartana, P.J., Wickwire, E.M. and Klick, B. (2010) Naturalistic Changes in Insomnia Symptoms and Pain in Temporomandibular Joint Disorder: A Cross-Lagged Panel Analysis. Pain, 149, 325-331. https://doi.org/10.1016/j.pain.2010.02.029

[8] Smith, M.T., Wickwire, E.M. and Grace, E.G. (2009) Sleep Disorders and Their Association with Laboratory Pain Sensitivity in Temporomandibular Joint Disorder. Sleep, 32, 779-790. https://doi.org/10.1093/sleep/32.6.779

[9] Cunali, P.A., Almeida, F.R. and Santos, C.D. (2009) Prevalence of Temporomandibular Disorders in Obstructive Sleep Apnea Patients Referred for Oral Appliance Therapy. Journal of Orofacial Pain, 23, 339-344.

[10] Edwards, R.R., Grace, E.S.P., Haythornthwaite, J.A. and Smith, M.T. (2009) Sleep Continuity and Architecture: Associations with Pain Inhibitory Processes in $\mathrm{Pa}$ tients with Temporomandibular Joint Disorder. European Journal of Pain, 13, 1043-1047. https://doi.org/10.1016/j.ejpain.2008.12.007

[11] Iber, C., Ancoli-Israel, S. and Chesson, A.L. (2007) The AASM Manual for the Scoring of Sleep and Associated Events: Rules, Terminology and Technical Specifications. American Academy of Sleep Medicine.

[12] International Classification of Sleep Disorders and American Academy of Sleep Medicine (2005) Diagnostic and Coding Manual. 2nd Edition.

[13] Jonas, D.E., Amick, H.R. and Feltner, C. (2017) Screening for Obstructive Sleep Apnea in Adults: Evidence Report and Systematic Review for the US Preventive Services Task Force. JAMA, 317, 415-433. https://doi.org/10.1001/jama.2016.19635 
[14] Guilleminault, C., Stoohs, R. and Clerk, A. (1993) A Cause of Excessive Daytime Sleepiness. The Upper Airway Resistance Syndrome. Chest, 104, 781-787. https://doi.org/10.1378/chest.104.3.781

[15] Stoohs, R.A., Knaack, L. and Blum, H.C. (2008) Differences in Clinical Features of Upper Airway Resistance Syndrome, Primary Snoring, and Obstructive Sleep Apnea/Hypopnea Syndrome. Sleep Medicine, 9, 121-128.

[16] Gold, A.R., Dipalo, F. and Gold, M.S. (2003) The Symptoms and Signs of Upper Airway Resistance Syndrome: A Link to the Functional Somatic Syndromes. Chest, 123, 87-95. https://doi.org/10.1378/chest.123.1.87

[17] Pépin, J.L., Guillot, M. and Tamisier, R. (2012) The Upper Airway Resistance Syndrome. Respiration, No. 83, 559-566. https://doi.org/10.1159/000335839

[18] Medicine AAoS (2014).

[19] Franklin, K.A., Sahlin, C. and Stenlund, H. (2013) Sleep Apnoea Is a Common Occurrence in Females. The European Respiratory Journal, 41, 615-619. https://doi.org/10.1183/09031936.00212711

[20] Li, K.K., Powell, N.B. and Guilleminauilt, C. (2000) Obstructive Sleep Apnea Syndrome: A Comparison between Far-East Asian and White Men. Laryngoscope, 110, 1689-1693. https://doi.org/10.1097/00005537-200010000-00022

[21] Khan, Y. and Heckmatt, J. (1994) Obstructive Apneas in Duchenne Muscular Dystrophy. Thorax, 49, 157-161. https://doi.org/10.1136/thx.49.2.157

[22] Kotagal, S., Gibbons, V. and Stith, J. (1994) Sleep Abnormalities in Patients with Severe Cerebral Palsy. Developmental Medicine \& Child Neurology, 36, 304-311. https://doi.org/10.1111/j.1469-8749.1994.tb11850.x

[23] Joosten, S.A., Hamilton, G.S. and Naughton, M.T. (2017) Impact of Weight Loss Management in OSA. Chest, 3692, 30157.

[24] Yousefian, J., Trimble, D. and DePaso, W. (2015) Correction of Severe Obstructive Sleep Apnea with Interdisciplinary Treatment. Dentitry Today, 34, 80-88.

[25] Yousefian, J. and Moghadam, B. (2013) The Role of Contemporary Orthodontics in the Diagnosis and Treatment of Sleep-Disordered Breathing. Roth Williams Legacy Foundation.

[26] Mihalko, W., Bergin, F., Kelly, F. and Canale, S.M. (2014) Obesity, Orthopaedics, and Outcomes. Journal of the American Academy of Orthopaedic Surgeons, 22, 683-690. https://doi.org/10.5435/JAAOS-22-11-683

[27] Piccin, C.F., Pozzebon, D. and Scapini, F. (2016) Craniocervical Posture in Patients with Obstructive Sleep Apnea. International Archives of Otorhinolaryngology, 20, 189-195. https://doi.org/10.1055/s-0036-1584295

[28] Sokucu, O., Oksayan, R. and Uyar, M. (2016) Relationship between Head Posture and the Severity of Obstructive Sleep Apnea. American Journal of Orthodontics and Dentofacial Orthopedics, 150, 945-949.

[29] Saito, M.L. (2013) Temporal Association between Sleep Apnea-Hypopnea and Sleep Bruxism Events. Journal of Sleep Research, 4.

[30] Raphael, K. and Masticatory, L.G. (2013) Muscle Sleep Background Electromyographic Activity Is Elevated in Myofascial Temporomandibular Disorder Patients. Journal of Oral Rehabilitation, 40, 883-891. https://doi.org/10.1111/joor.12112

[31] Prinsell, J. (2015) Telegnatic Maxillomandibular Advancement Surgery for OSA. Dental Sleep Practice, 6-9.

[32] Yousefian, J. (2016) Correction of Severe Obstructive Sleep Apnea Syndrome with 
Interdisciplinary Medical and Dental Treatment Planning. Journal of Dental Sleep Medicine, 3, 1-3. https://doi.org/10.15331/jdsm.5994

[33] Wang, Q., Jia, P. and Nk, A. (2012) Changes of Pharyngeal Airway Size and Hyoid Bone Position Following Orthodontic Treatment of Class I Bimaxillary Protrusion. Angle Orthodontist, 82, 115-121. https://doi.org/10.2319/011011-13.1

[34] Germec-Cakan, D., Taner, T. and Akan, S. (2011) Uvulo-Glossopharyngeal Dimensions in Non-Extraction, Extraction with Minimum Anchorage, and Extraction with Maximum Anchorage. European Journal of Orthodontics, 33, 515-520. https://doi.org/10.1093/ejo/cjq109

[35] Paul, S.A., Simon, S.S. and Issac, B. (2015) Management of Severe Sleep Apnea Secondary to Juvenile Arthritis with Temporomandibular Joint Replacement and Mandibular Advancement. Journal of Pharmacy and Bioallied Sciences, 687-690. https://doi.org/10.4103/0975-7406.163479

[36] Wolford, L.M., Mercuri, L.G. and Schneiderman, E.D. (2015) Twenty-Year Follow-Up Study on a Patient-Fitted Temporomandibular Joint Prosthesis: The Techmedica/TMJ Concepts Device. Journal of Oral and Maxillofacial Surgery, 73, 952-960.

[37] Conley, S. and Legan, H. (2006) Correction of Severe Obstructive Sleep Apnea with Bimaxillary Transverse Distraction Osteogenesis and Maxillomandibular Advancement. American Journal of Orthodontics and Dentofacial Orthopedics, 129, 283-292.

[38] Kunkel, M.H.W. (1997) The Influence of Maxillary Osteotomy on Nasal Airway Patency and Geometry. Mund-Kiefer-Gesichtschirurgie, 94-98.

https://doi.org/10.1007/BF03043550

[39] Courtiss, E. and Goldwyn, R. (1983) The Effects of Nasal Surgery on Airflow. Plastic and Reconstructive Surgery, 72, 9-21. https://doi.org/10.1097/00006534-198307000-00003

[40] Yousefian, J., Trimble, D. and Folkman, G. (2006) A New Look at the Treatment of Class II Division 2 Malocclusion. American Journal of Orthodontics and Dentofacial Orthopedics, 130, 771-778.

[41] Sarver, D. (2001) The Importance of Incisor Positioning in the Esthetic Smile: The Smile Arc. American Journal of Orthodontics and Dentofacial Orthopedics, 120, 98-111. https://doi.org/10.1067/mod.2001.114301

[42] Sarver, D.A.M. (2003) Dynamic Smile Visualization and Quantification: Part 2 (Smile Analysis and Treatment Strategies). American Journal of Orthodontics and Dentofacial Orthopedics, 124, 116-127.

[43] Peck, S.P.L. (1995) Selected Aspects of the Art and Science of Facial Esthetics. Seminars in Orthodontics, 1, 105-126.

[44] Zachrisson, B.U. (1998) Esthetic Factors Involved in Anterior Tooth Display and the Smile, Vertical Dimension. Journal of Clinical Orthodontics, 32, 432-445.

[45] Yousefian, J. and Moghadam, B. (2015) The Role of Contemporary Orthodontics in the Diagnosis and Treatment of Sleep-Disordered Breathing. 2nd Edition, Roth Williams Legacy Foundation. 